\title{
Magnetic field in relativistic heavy-ion collisions: Testing the classical approximation
}

\author{
I. Danhoni $\odot$ and F. S. Navarra \\ Instituto de Física, Universidade de São Paulo, Rua do Matão, 1371, CEP 05508-090, São Paulo, SP, Brazil
}

(Received 10 November 2020; accepted 19 January 2021; published 2 February 2021)

\begin{abstract}
It is believed that in noncentral relativistic heavy-ion collisions a very strong magnetic field is formed. There are several studies of the effects of this field, where $\vec{B}$ is calculated with the expressions of classical electrodynamics. A quantum field may be approximated by a classical one when the number of field quanta in each field mode is sufficiently high. This may happen if the field sources are intense enough. In heavy-ion physics the validity of the classical treatment was not investigated. In this work we propose a test of the quality of the classical approximation. We calculate an observable quantity using the classical magnetic field and also using photons as input. If the results of both approaches coincide, this will be an indication that the classical approximation is valid. More precisely, we focus on the process in which a nucleon is converted into a delta resonance, which then decays into another nucleon and a pion, i.e., $N \rightarrow \Delta \rightarrow N^{\prime} \pi$. In ultraperipheral relativistic heavy-ion collisions this conversion can be induced by the classical magnetic field of one of the ions acting on the other ion. Alternatively, we can replace the classical magnetic field by a flux of equivalent photons, which are absorbed by the target nucleons. We calculate the cross sections in these two independent ways and find that they differ from each other by $\simeq 10 \%$ in the considered collision energy range. This suggests that the two formalisms are equivalent and that the classical approximation for the magnetic field is reasonable.
\end{abstract}

DOI: 10.1103/PhysRevC.103.024902

\section{INTRODUCTION}

It was often said that in relativistic heavy-ion collisions we produce the strongest magnetic field of the universe [1-3]. This field is so intense because the charge density is large, because the speed of the source is very close to the speed of light and also because we probe it at extremely small distances (a few Fermi) from the source.

There was a search for observable effects of this strong field [4]. The first and most famous is the chiral magnetic effect (CME) [5]. A natural place to look for this field and its effects is in ultraperipheral relativistic heavy-ion collisions (UPCs), in which the two nuclei do not overlap [6]. Because there is no superposition of hadronic matter, the strong interaction is suppressed and the collision becomes essentially a very clean electromagnetic process.

In [7] it was argued that forward pions are very likely to be produced by magnetic excitation (ME) of the nucleons in the nuclei. The strong classical magnetic field produced by one nucleus induces magnetic transitions, such as $N \rightarrow \Delta$ (where $N$ is a proton or a neutron), in the nucleons of the other nucleus. The produced $\Delta$ keeps moving together with the nucleus (or very close to it) and then decays almost exclusively

Published by the American Physical Society under the terms of the Creative Commons Attribution 4.0 International license. Further distribution of this work must maintain attribution to the author $(s)$ and the published article's title, journal citation, and DOI. Funded by SCOAP . through the reaction $\Delta \rightarrow N+\pi$. From the kinematics we know that the pion has a very large longitudinal momentum and very large rapidity. Because there is no other competing mechanism for forward pion production in UPCs the observation of these pions would be a signature of the magnetic excitation of the nucleons and also an indirect measurement of the magnetic field. In [7] it was shown that ME has a very large cross section.

The hypothesis that in heavy-ion collisions the electromagnetic field can be treated classically and one can speak of a classical magnetic field has never been tested. The classical field approximation may be expected to become a reliable description of the quantum theory if the number of field quanta in each field mode is sufficiently high. In this work we propose a way to test the classical approximation for the magnetic field. To this end we consider again the process discussed in [7]

$$
N \rightarrow \Delta \rightarrow N^{\prime} \pi
$$

but this time the transition is induced by photons and not by the classical magnetic field. We compute the same process using a different formalism where the quanta of the field play the important role. We then compare the results obtained with the two formalisms. To avoid uncertainties associated with the spatial distribution of the nuclear matter in the target we consider lead-proton collisions and choose to work in the proton rest frame.

In the next section we briefly review the formalism used in [7] which we shall call semiclassical and in the following section we describe the quantum formalism, based on the 


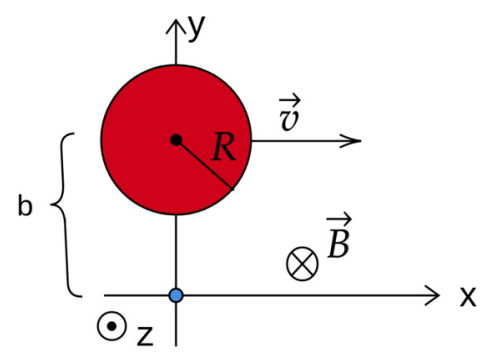

FIG. 1. $P b-p$ collision in the proton rest frame. Coordinate system with magnetic field along the $z$ direction. The projectile nucleus of radius $R$ moves with velocity $\vec{v}$ and impact parameter $b$. The blue circle represents the proton at rest.

equivalent photon approximation. In the end we compare the results obtained with the two methods.

\section{THE SEMICLASSICAL FORMALISM}

A strong magnetic field can convert a hadron into another one with a different spin, by "flipping the constituent quark spins." In ultrarelativistic heavy-ion collisions this idea was first advanced in [8], where the authors studied the transition $\eta_{c} \rightarrow J / \psi$. In [7] we extended the calculations to the $N \rightarrow \Delta$ transition.

Let us consider an ultraperipheral $\mathrm{Pb}-p$ collision, where the proton is at rest, as shown in Fig. 1. Under the influence of the strong magnetic field generated by the moving nucleus, the nucleon is converted into a $\Delta$. For the sake of definiteness let us consider the transition $|p \uparrow\rangle \rightarrow\left|\Delta^{+} \uparrow\right\rangle$. The amplitude for this process is given by $[7,8]$

$$
a_{f i}=-i \int_{-\infty}^{\infty} e^{i E_{f i} t^{\prime}}\left\langle\Delta^{+} \uparrow\left|H_{\mathrm{int}}\left(t^{\prime}\right)\right| p \uparrow\right\rangle d t^{\prime},
$$

where $\hbar=1$ and $E_{f i}=\left(m_{\Delta}^{2}-m_{n}^{2}\right) / 2 m_{n}$, where $m_{\Delta}$ and $m_{n}$ are the $\Delta$ and nucleon masses respectively. The interaction Hamiltonian is given by

$$
H_{\mathrm{int}}(t)=-\vec{\mu} \cdot \vec{B}(t)
$$

The magnetic dipole moment of the nucleon is given by the sum of the magnetic dipole moments of the corresponding constituent quarks:

$$
\vec{\mu}=\sum_{i=u, d} \vec{\mu}_{i}=\sum_{i=u, d} \frac{q_{i}}{m_{i}} \vec{S}_{i}
$$

where $q_{i}$ and $m_{i}$ are the charge and constituent mass of the quark of type $i$ and $\vec{S}_{i}$ is the spin operator acting on the spin state of this quark. In Fig. 1 we show the system of coordinates and the moving projectile. The projectile of radius $R$ moves along the $x$ direction with impact parameter $b$ and the magnetic field is in the $z$ direction. Because we are studying a UPC, we will, for simplicity, assume that the projectilegenerated field is the same produced by a point charge. The field is given by [3]

$$
B_{z}(t)=\frac{1}{4 \pi} \frac{q v \gamma(b-y)}{\left((\gamma(x-v t))^{2}+(y-b)^{2}+z^{2}\right)^{3 / 2}} .
$$

In the above expression $\gamma$ is the Lorentz factor, $b$ is the impact parameter along the $y$ direction, $v \simeq 1$ is the projectile velocity, and the projectile electric charge is $q=Z e$.

The interaction Hamiltonian acts on spin states. The relevant ones are

$$
\begin{aligned}
|p \uparrow\rangle= & \frac{1}{3 \sqrt{2}}[u d u(\downarrow \uparrow \uparrow+\uparrow \uparrow \downarrow-2 \uparrow \downarrow \uparrow) \\
& +d u u(\uparrow \downarrow \uparrow+\uparrow \uparrow \downarrow-2 \downarrow \uparrow \uparrow) \\
& +u u d(\uparrow \downarrow \uparrow+\downarrow \uparrow \uparrow-2 \uparrow \uparrow \downarrow)], \\
\left|\Delta^{+} \uparrow\right\rangle= & \frac{1}{3}(u u d+u d u+d u u)(\uparrow \uparrow \downarrow+\uparrow \downarrow \uparrow+\downarrow \uparrow \uparrow) .
\end{aligned}
$$

With these ingredients we can compute the matrix element $\left\langle\Delta^{+} \uparrow\left|H_{\text {int }}\right| p \uparrow\right\rangle$. It can be obtained by substituting Eqs. (4) and (5) into Eq. (3) and then calculating the sandwiches of $H_{\text {int }}$ with the spin states given above. Evaluating the nucleon-delta transition matrix element we find

$$
\left\langle\Delta^{+} \uparrow\left|H_{\mathrm{int}}\right| p \uparrow\right\rangle=\frac{\sqrt{2} e}{3 m} B_{z} .
$$

The cross section for a single $N \rightarrow \Delta$ transition is given by

$$
\sigma=\int\left|a_{f i}\right|^{2} d^{2} b=2 \pi \int\left|a_{f i}\right|^{2} b d b,
$$

where we have used cylindrical symmetry $d^{2} b=b d b d \theta \rightarrow$ $2 \pi b d b$. Inserting (8) into (2) and using it in the above expression we find

$$
\sigma=\frac{Z^{2} e^{4}}{9 \pi m^{2}}\left(\frac{E_{f i}}{v \gamma}\right)^{2} \int_{R}^{\infty}\left[K_{1}\left(\frac{E_{f i} b}{v \gamma}\right)\right]^{2} b d b,
$$

where $K_{1}$ is the modified Bessel function. This is the result obtained with the semiclassical approach. For the purpose of this work it is enough to consider a nucleon as a target. In [7] we computed the cross section for a nucleus-nucleus collision.

\section{THE QUANTUM FORMALISM}

In the quantum formalism, the electromagnetic field produced by an ultrarelativistic electric charge is replaced by a flux of photons [6]. Now, in a high energy UPC, the projectile becomes a source of almost real photons and we replace the classical field by a collection of quanta. Thus, the cross section of the process (1) can be written in a factorized form in terms of the photon flux produced by the projectile and the photon-nucleon cross section [6]:

$$
\sigma=\int \frac{d \omega}{\omega} n(\omega) \sigma_{\gamma N \rightarrow N \pi}(\omega) .
$$

In the above expression $n(\omega)$ represents the photon spectrum generated by the source [6]:

$$
\begin{aligned}
n(\omega) & =\frac{Z^{2} \alpha}{\pi}\left[2 \xi K_{0}(\xi) K_{1}(\xi)-\xi^{2}\left[K_{1}^{2}(\xi)-K_{0}^{2}(\xi)\right]\right], \\
\xi & =\frac{\omega\left(R_{1}+R_{2}\right)}{\gamma},
\end{aligned}
$$

where $\omega$ is the photon energy, $R_{1}$ and $R_{2}$ are the radii of the projectile and the target, parametrized as $R_{A}=1.2 A^{1 / 3} \mathrm{fm}$, 


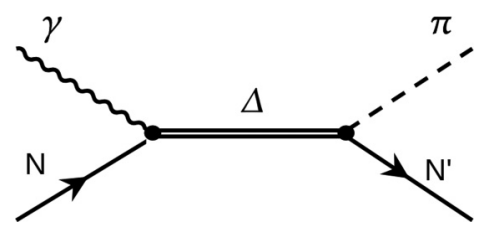

FIG. 2. Quantum version of the process depicted in Fig. 1. Pion photoproduction through a $\Delta$ resonance.

and $\gamma$ the Lorentz boost in the target frame. From the above expression it is clear that the average energy carried by an emitted photon increases with $\gamma$ and hence with the collision energy $\sqrt{s}$. The photon average energy may be estimated as

$$
\bar{\omega}=\frac{\int_{0}^{\gamma m_{n}} n(\omega) \omega d \omega}{\int_{0}^{\gamma m_{n}} n(\omega) d \omega} .
$$

In the LHC energy region $\gamma \simeq 1000$ and the above expression yields $\bar{\omega} \simeq 10 \mathrm{GeV}$.

To perform the calculation of the total cross section, it is necessary to know the cross section of the process $\gamma N \rightarrow N \pi$. In a first approximation $\sigma_{\gamma N \rightarrow N \pi}$ can be calculated evaluating the Feynman diagram shown in Fig. 2. This is a very well-known process. In fact, there is an intense effort devoted to the study of nucleon resonances both experimentally and theoretically [9-14]. Most of the interest lies on the energy region around the threshold of $\Delta$ production, i.e., $\bar{\omega} \simeq 200$ $600 \mathrm{MeV}$. As it was just mentioned above, we are primarily interested in the high energy region, far from this threshold. We need a formula which correctly reproduces the behavior of the cross section in the $\Delta$ resonance region and which can be extrapolated to higher energies. This is the most important source of uncertainty in the evaluation of (11).

A simple parametrization of the $\pi^{0}$ photoproduction cross section can be taken from Jones and Scadron [15]:

$$
\begin{aligned}
\sigma_{\gamma N \rightarrow N \pi}(\omega)= & 2 \pi \int_{0}^{\pi} d \theta \sin \theta \frac{\alpha \omega}{12 m_{n} W} \\
& \times \frac{\sin ^{2} \delta}{\Gamma}\left[\left|F_{+}^{*}\right|^{2} f(\theta)+\left|G_{+}^{*}\right|^{2} g(\theta)\right] .
\end{aligned}
$$

In the above expression $\alpha=1 / 137, W^{2}=m_{n}^{2}+2 \omega m_{n}$ is the photon-nucleon center-of-mass energy squared, $m_{n}$ is the nucleon mass, $\Gamma$ is the $\Delta$ decay width, and $F_{+}^{*}=G_{M}^{*}-3 G_{E}^{*}$, $G_{+}^{*}=G_{M}^{*}+G_{E}^{*}$. The form factors $G_{M}^{*}$ and $G_{E}^{*}$ are functions of the photon virtuality $Q^{2}$. Because we are interested in photoproduction they are taken at $Q^{2}=0$. The calculations were all carried out in the laboratory frame. The angular dependence is given by $f(\theta)=\left(3 \cos ^{2} \theta+1\right) / 2, g(\theta)=$ $\left(9 \sin ^{2} \theta\right) / 2$ and

$$
\sin ^{2} \delta=\left|\frac{m_{\Delta} \Gamma}{\left(W^{2}-m_{\Delta}^{2}+i m_{\Delta} \Gamma\right)}\right|^{2} .
$$

The expression (14) contains three parameters $G_{M}^{*}, G_{E}^{*}$, and $\Gamma$, which can be determined by fitting the experimental data on $\pi^{0}$ photoproduction. We have adjusted (14) to the data published in [16]. The result is shown in Fig. 3. To estimate

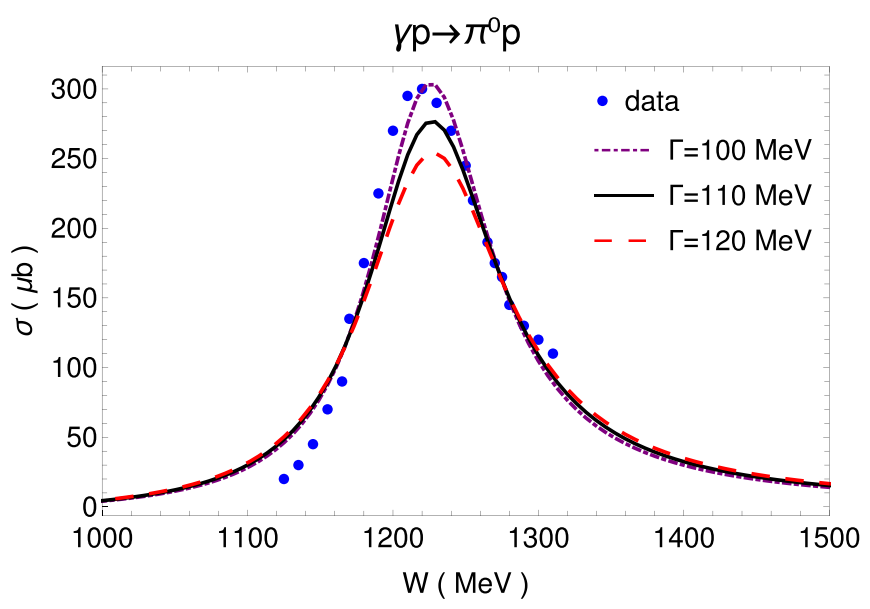

FIG. 3. Pion photoproduction. Comparison between the theory, Eq. (14), and data from [16].

the uncertainty in the extrapolation of (14) to higher photon energies we have varied the $\Delta$ width within the interval $100<\Gamma<120 \mathrm{MeV}$. As it can be seen, the high energy tail of the curve is not very sensitive to changes in $\Gamma$. The uncertainties in $G_{M}^{*}(0)$ and $G_{E}^{*}(0)$ are very small and changes of these quantities would not significantly change the cross sections.

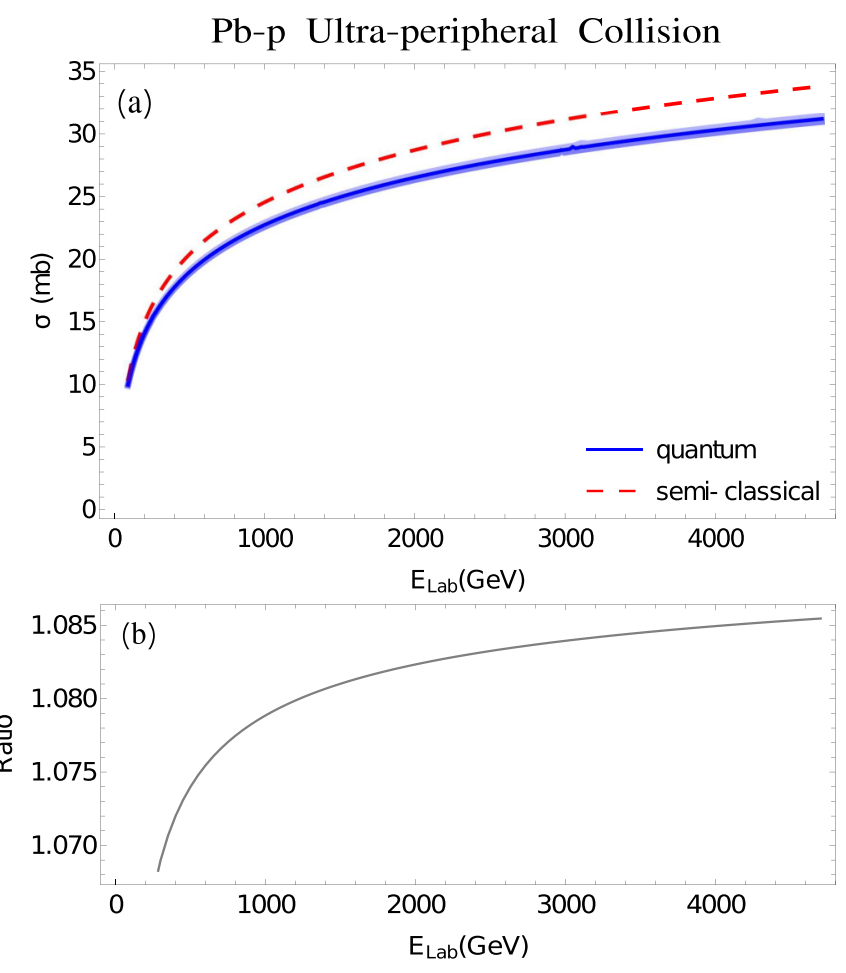

FIG. 4. Comparison between the semiclassical and quantum cross sections of pion photoproduction in an ultraperipheral leadnucleon collision. (Upper panel) Cross sections as a function of the energy per nucleon in the laboratory frame. (Lower panel) Ratio between semiclassical and quantum cross sections. 
Having determined $\sigma_{\gamma N \rightarrow N \pi}$, we insert it into (11) and evaluate the cross section of the quantum process. The results are then compared with the results obtained with the semiclassical approach [given by (10)] and presented in Fig. 4. The cross sections are plotted as a function of the energy per nucleon (of the projectile) in the laboratory frame $E_{\mathrm{Lab}}=\gamma m_{n}$. In the upper panel we compare the curves obtained with (10) (dashed line) and with (11) (solid lines). The band in the lower curve represents the different choices of the width $\Gamma$, i.e., the different values shown in Fig. 3. In the lower panel we show (10) divided by the central value of (11). This ratio quantifies the difference between these two curves and it approaches $9 \%$ at the highest energies. These results suggest that the classical approximation of the magnetic field reproduces most of the photon interaction in photoproduction in high energies.

The quantum formula (14) could be improved. At low energies there are other resonances. To improve the accuracy of the extrapolation to higher photon energies, it would be necessary to change (14) including higher resonances or, alternatively, define some procedure to "average over the bumps," as it was done (although in a different context), for example, in [17].

\section{CONCLUDING REMARKS}

In heavy-ion collisions the sources are so intense that one can treat classically the electromagnetic field. In particular, one can compute the magnetic field and use it to make a number of predictions. Although plausible, this conjecture had never been tested before. In this work we have devised a test for this idea. We have found a process which can be calculated in two different ways: one using the magnetic field and one relying solely on quantum physics. The EPA method was extensively used and has yielded predictions confirmed by experimental data.

Our results give some support to the classical approximation for the magnetic field and hence give support to all the calculations done previously based on this approximation.

\section{ACKNOWLEDGMENTS}

The authors are deeply grateful to G. Ramalho for very instructive discussions. This work was partially financed by the Brazilian funding agencies CAPES and CNPq.
[1] V. Skokov, A. Y. Illarionov, and V. Toneev, Int. J. Mod. Phys. A 24, 5925 (2009).

[2] V. Voronyuk, V. D. Toneev, W. Cassing, E. L. Bratkovskaya, V. P. Konchakovski, and S. A. Voloshin, Phys. Rev. C 83, 054911 (2011).

[3] M. Asakawa, A. Majumder, and B. Muller, Phys. Rev. C 81, 064912 (2010).

[4] For a recent review see K. Hattori and X. G. Huang, Nucl. Sci. Tech. 28, 26 (2017), and references therein; A. Dubla, U. Gürsoy, and R. Snellings, Modern Phys. Lett. A 35, 2050324 (2020); C. S. Machado, F. S. Navarra, E. G. de Oliveira, J. Noronha, and M. Strickland, Phys. Rev. D 88, 034009 (2013).

[5] K. Fukushima, D. E. Kharzeev, and H. J. Warringa, Phys. Rev. D 78, 074033 (2008).

[6] C. A. Bertulani, S. R. Klein, and J. Nystrand, Ann. Rev. Nucl. Part. Sci. 55, 271 (2005); V. P. Goncalves and M. V. T. Machado, Eur. Phys. J. C 40, 519 (2005).

[7] I. Danhoni and F. S. Navarra, Phys. Lett. B 805, 135463 (2020).

[8] D. L. Yang and B. Muller, J. Phys. G 39, 015007 (2012).
[9] For a very recent and comprehensive theoretical and experimental review, see D. G. Ireland, E. Pasyuk, and I. Strakovsky, Prog. Part. Nucl. Phys. 111, 103752 (2020) and references therein.

[10] V. Pascalutsa, M. Vanderhaeghen, and S. N. Yang, Phys. Rept. 437, 125 (2007).

[11] I. G. Aznauryan et al. (CLAS Collaboration), Phys. Rev. C 80, 055203 (2009).

[12] I. G. Aznauryan and V. D. Burkert, Prog. Part. Nucl. Phys. 67, 1 (2012)

[13] G. H. G. Navarro and M. J. Vicente Vacas, Phys. Rev. D 102, 113016 (2020).

[14] G. Ramalho, Phys. Rev. D 100, 114014 (2019); Eur. Phys. J. A 55, 32 (2019); 54, 75 (2018); Phys. Rev. D 94, 114001 (2016).

[15] H. F. Jones and M. D. Scadron, Annals Phys. 81, 1 (1973).

[16] D. A. McPherson, D. C. Gates, R. W. Kenney, and W. P. Swanson, Phys. Rev. 136, B1465 (1964); M. MacCormick et al., Phys. Rev. C 53, 41 (1996).

[17] S. J. Brodsky and F. S. Navarra, Phys. Lett. B 411, 152 (1997). 